\title{
Design and Performance of the ISS-CREAM Boronated Scintillator Detector
}

\author{
Jason Link ${ }^{1}$ (for the ISS-CREAM Collaboration ${ }^{2}$ ) \\ NASA Goddard Space Flight Center \\ 8800 Greenbelt Road, Greenbelt Maryland 20771 \\ E-mail: Jason.Link@nasa.gov
}

\begin{abstract}
Cosmic Ray Energetics And Mass for the International Space Station (ISS-CREAM) is a new instrument developed to measure the composition and spectrum of cosmic-ray particles up to close to the knee of the cosmic-ray energy spectrum $\left(10^{12}-10^{15} \mathrm{eV}\right)$. The instrument utilizes two modified detectors from the highly successful CREAM balloon instrument, a sampling calorimeter and silicon charge detector. Two new detectors systems, the Top and Bottom Counting Detector (T/BCD) and Boronated Scintillator Detector (BSD) have been built to enable making measurements of high-energy electrons in the instrument. In this paper we focus on the Boronated Scintillator Detector, which detects thermal neutrons in the showers produced by cosmic rays interacting in the calorimeter. We can discriminate between hadrons and electrons by looking at the number of thermal neutrons produced in these showers. A larger yield of neutrons is produced from hadronic interaction by cosmic-ray nuclei than electromagnetic interactions by high-energy cosmic-ray electrons in showers that deposit equal amounts of energy in the calorimeter. The BSD consists of a detector enclosure containing the boronated scintillator and photomultiplier tubes and an electronic enclosure with signal conditioning, control, communication, housekeeping and power conditioning electronics. We present data on the performance of the detector as measured in the lab and at the CERN H2 beamline in 2012.
\end{abstract}

The 34th International Cosmic Ray Conference

30 July- 6 August , 2015

The Hague, The Netherlands

\footnotetext{
${ }^{1}$ Speaker

${ }^{2}$ http://cosmicray.umd.edu/iss-cream/collaboration 


\section{Introduction}

The ISS-CREAM experiment is an evolution of the CREAM balloon program utilizing previously developed detectors and technologies. The scientific goal of the CREAM program is to measure the composition and energy spectrum of elements between $\mathrm{Z}=1$ and $\mathrm{Z}=26$ at energies between $10^{12} \mathrm{eV}$ and $10^{15} \mathrm{eV}$. The total energy spectrum of all cosmic rays can be expressed by a power law, but around $10^{15} \mathrm{eV}$ the power index of this spectrum changes from $\sim-2.7$ to $\sim-3.1$. Measurements of individual elemental energy spectra around this energy may yield clues to what is causing this spectral break and add to our understanding of the source and acceleration mechanism behind cosmic rays. ISS-CREAM [1] will fly two detectors modified and enhanced from the CREAM balloon program to perform this measurement from the International Space Station (ISS), a sampling calorimeter and a silicon pixel charge detector (SCD). In addition, ISS-CREAM will fly three new detectors, the Top and Bottom Counting Detectors and the Boronated Scintillator Detector, which will enable a new science goal of measuring the cosmicray electron spectrum above $100 \mathrm{GeV}$. This measurement will allow for a probe of any local sources for cosmic rays as electrons lose energy quickly propagating in interstellar space. Data from the BSD, BCD and TCD in conjunction with data from the sampling calorimeter allow for discrimination between electrons and protons which are otherwise indistinguishable by the SCD and sampling calorimeter alone.

\section{Detector Considerations}

A cosmic-ray proton and an electron with the same initial energy that interact in the ISS-CREAM calorimeter will be detected as events with the same charge but will deposit different amounts of energy in the calorimeter; the proton will deposit $~ 1 / 3$ of the amount of energy of that of the electron. Additionally, the hadron will produce roughly an order magnitude more neutrons than the electron in its interaction shower. These neutrons will thermalize in the calorimeter over the course of several microseconds. The BSD takes advantage of the presence of these late neutrons and detects them after the charged particles from the initial shower have passed through the detector. By combining this neutron signal with the energy measured by the calorimeter we can determine whether a particle is an electron or proton.

The BSD consists of a $60 \mathrm{~cm}$ x $60 \mathrm{~cm}$ x $3.8 \mathrm{~cm}$ block of Eljen EJ-254 scintillator which is loaded with $5 \%$ boron concentration by weight [2]. Neutrons entering into the scintillator interact with the boron via the reaction:

$$
{ }^{10} \mathrm{~B}+n \rightarrow L i^{3}+\alpha+\gamma
$$

Each thermal neutron capture produces $\sim 570$ photons. The capture probability of a neutron in the BSD is a decaying exponential with time constant $\tau$ given by

$$
\tau=\left(N\left({ }^{10} B\right) \sigma V\right)^{-1}
$$

where $N\left({ }^{10} \mathrm{~B}\right)$ is the number density of $\mathrm{B}, \sigma$ is the cross section for capture and $V$ is the neutron velocity. For low energy neutrons $\left(E_{n}<200 \mathrm{keV}\right)$ we find $t=2.74 \mu \mathrm{s}$. In order to maximize our neutron signal we will utilize charge integrating analog-to-digital converters (ADCs) active from about $1 \mu$ s to $6 \mu$ s (described below) to collect as much light as possible from the neutrons. 
The BSD consists of two enclosures, one for the scintillator and photomultiplier tubes and one for the readout and detector electronics. The detector enclosure, shown in Figures 1a and 1c, consists of a box body machined from a single piece of aluminum and a gasketed lid. The scintillator sits in the center of the detector enclosure surrounded on 4 sides by polyurethane foam which cushions the scintillator from vibration and shock during launch. The scintillator is wrapped in Teflon (not shown in Figure 1a) to protect the surface and redirect light that escapes total internal reflection inside the plastic. On two sides of the scintillator 1" R1924A Hammamatsu PMTs look into the scintillator to detect light from particles interacting in the plastic. Two of these PMTs are for triggering. These are air coupled to the scintillator and provide a trigger to the flight electronics. Sixteen of the PMTs are equipped with a rapid highvoltage switching circuit (described below), and are packaged mechanically and electronically in sets of four (visible in Figure 1a). We refer to these as quartet PMT units. The quartet PMT units are optically coupled via a piece of acrylic and Nusil CV4-2500 epoxy directly to the scintillator and measure photons from neutron interactions.
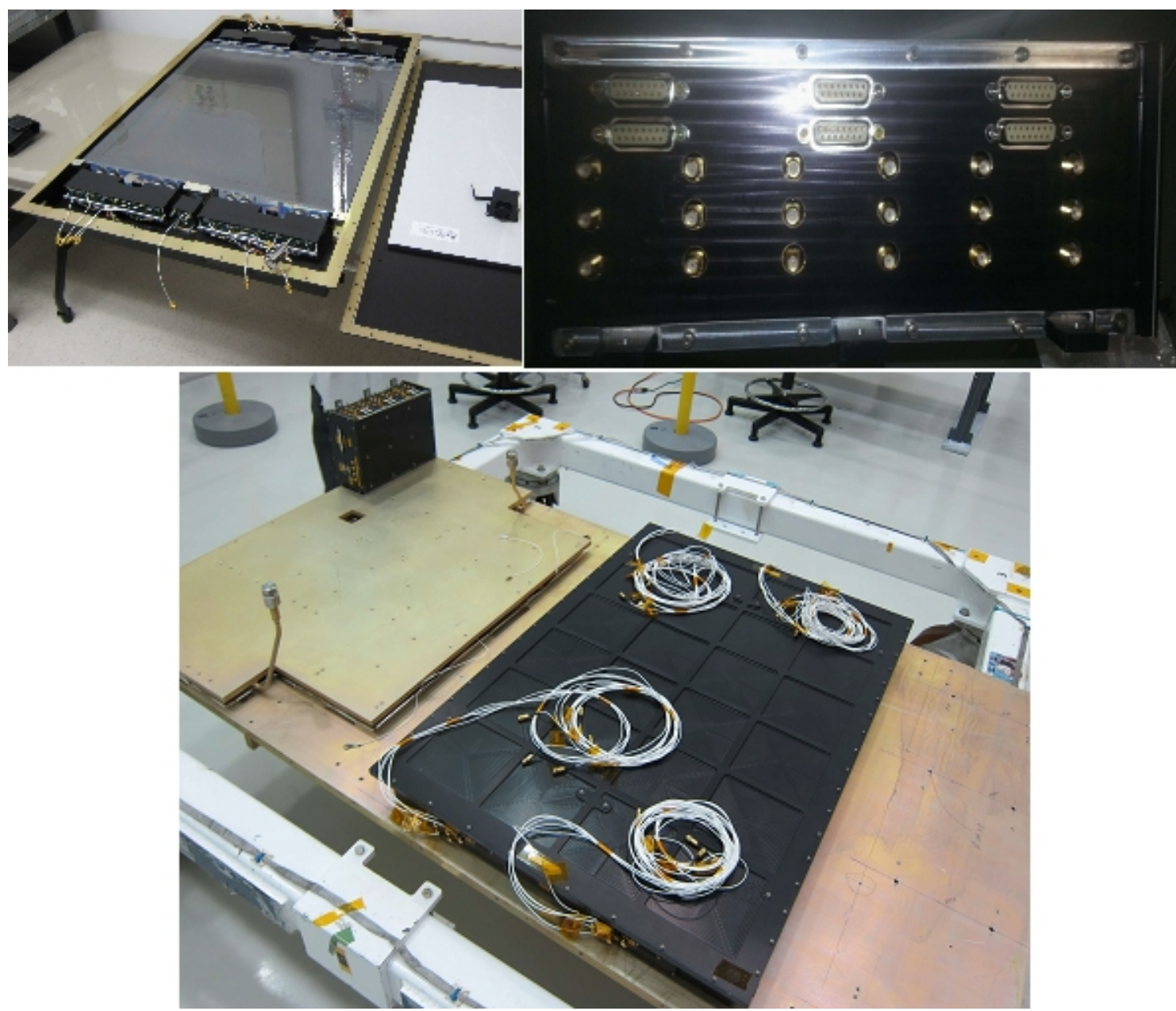

Figure 1: Photos of the BSD Flight Hardware. Upper Left (1a) Detector enclosure being assembled. Upper Right (1b) The electronics enclosure prior to cabling for TV testing. Lower (1c) Detector and electronic enclosure being mechanically integrated to the ISS-CREAM baseplate at the NASA Wallops Flight Facility. 
Early on in our development process we determined through a series of laboratory tests that there was an issue detecting the post-shower neutron signal with a standard PMT base design. The light in the scintillator produced by charged particles from the particle shower saturates the PMTs creating an afterpulse which is much larger than the post-shower neutron signal itself. The solution we adopted is to utilize a switched dynode design where we switch power to the first two dynodes of the quartet PMTs approximately 100 ns after we detect the charged particle shower passing through the scintillator with our trigger PMTs. We then allow 900 ns to pass for the switching transient of the HV to occur and the PMTs to settle before we start our gated integration of the neutron signal. The timing diagram of this process is shown in Figure 2. The trigger PMTs are not switched but instead made with a tapered base design, similar to one utilized for the SuperTIGER experiment [3]. The signal from each of the trigger tubes is split off to a discriminator circuit to provide an internal detector trigger. The signal from the trigger tubes is digitized in a much smaller sampling window of a few hundred nanoseconds immediately after a threshold is crossed, providing us with a measurement of the interaction shower which may be useful in analysis.

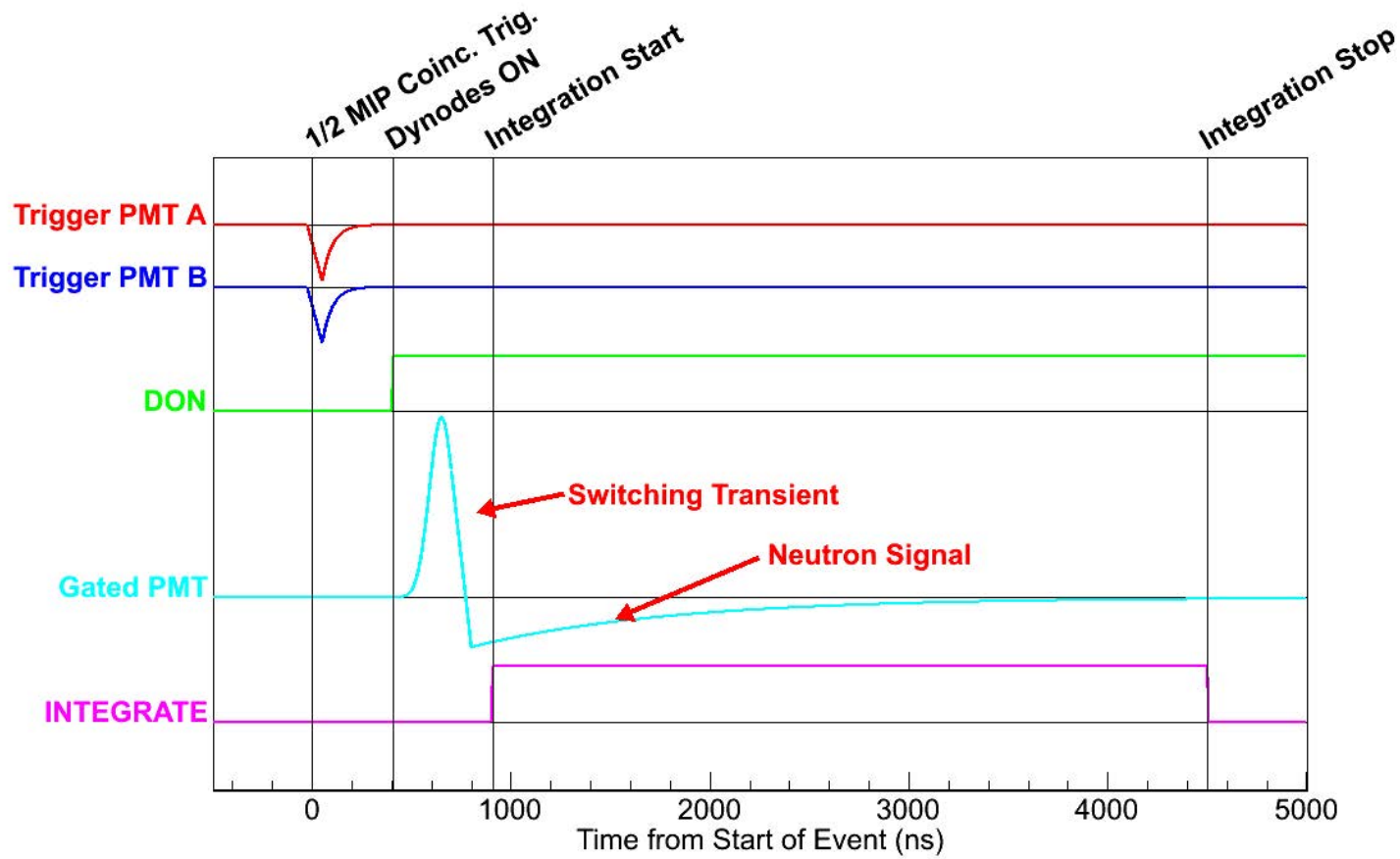

Figure 2: BSD Electronics Timing Diagram. The interaction shower from the cosmic ray interacting in the calorimeter causes a trigger to form by the signal in the two trigger PMTs. This initiates a switch (DON) to turn on power to the dynodes D0 and D1 of all quartet (gated) PMTs. Several hundred nanoseconds later, after the switching transient from turning on D0 and D1, integration begins.

The BSD electronics enclosure, shown in Figures 1b and 1c, contains the digitization and readout, PMT control, USB communication, power conditioning, triggering and housekeeping electronics that run the BSD detector. This functionality is distributed on 5 boards which are stacked on $1 / 2$ " standoffs inside the enclosure box. Three of these boards have a common design and are known as the Front End Electronic (FEE) boards. The FEE boards digitize PMT signals from 6 tubes utilizing a 16 bit ADC. Each channel has two different gain branches, which 
allows for resolution of signals as small as 1 photoelectron from the R1924A PMT on the high gain branch, while the low gain branch allows for the measurement of signals up to $\sim 8 \times 10^{7}$ photoelectrons. The FEE boards also have one-shot discrimination circuitry on one channel which enables the formation of an event trigger which initiates powering up the first two dynodes of the quartet PMTs and the data digitization sequence. The functionality of the BSD as well as communication with the ISS-CREAM payload is handled by the control board. Communication with the ISS-CREAM science flight computer is handled via USB with two independent FX2lP microcontrollers on the control board providing a redundant communication pathway. The control board also houses an A3P1000 FPGA which manages all communication and control of the BSD detector system including event processing and packaging, controlling the high voltage supplies levels of the BSD PMTs as well as the dynode switching on the quartet PMTs, and setting discrimination levels for triggers and scalars. Finally the housekeeping and power board receives power in from the ISS-CREAM common electronics and conditions it to the voltage levels required by the BSD electronics and PMT assemblies. The board also has circuitry to condition and package voltage, current, and temperature housekeeping information to distribute to the ISS-CREAM common electronics. Finally it has a pulser circuit which drives two LEDs inside the detector enclosure which are used to test the PMTs.

\section{Detector Testing and Performance}

Early on in the BSD development we had the opportunity to take our boronated scintillator and some prototype PMT assemblies to CERN. We were able to test the hardware in both a pion and electron beam to verify the design and performance of the BSD. The boronated scintillator was placed behind the CREAM sampling calorimeter and exposed to a pion beam between 100-175 GeV and an electron beam between 250-350 GeV, We were able to clearly see signals in the detector between 900 ns and $5 \mu$ s after the particle interaction in the calorimeter which we determined could only be from neutrons. Furthermore as can be seen in Figure 3, when we compare the signal distribution in the calorimeter and the BSD, we can clearly see the ability of the BSD to distinguish hadrons from electrons.
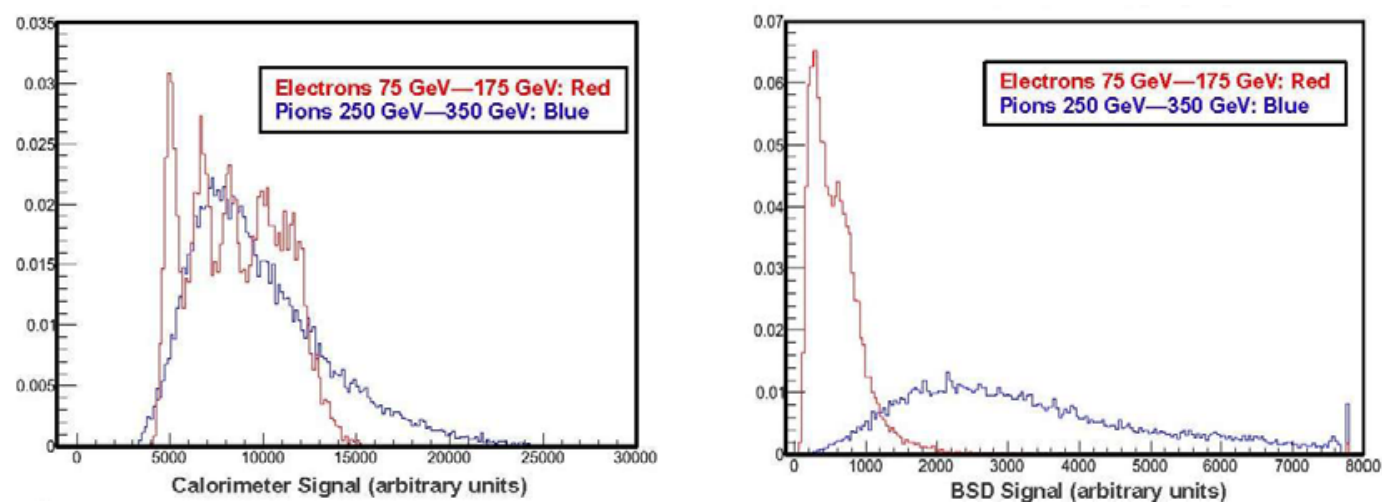

Figure 3: Calorimeter (left) and BSD (right) signals for electron and pion runs at CERN in November 2012. Electrons runs (red) were at 75, 100, 125, 150 and $175 \mathrm{Gev.} \mathrm{Pion} \mathrm{runs} \mathrm{(blue)}$ were at 250, 300 and $350 \mathrm{GeV}$. The histograms count (y-axis) is normalized by the total number of pion or electron events during the runs. 
We have quantified the discrimination ability of the BSD using the CERN data to calculate the rejection power of our detector, defined here as:

$$
\text { Rejection Power }=\frac{\text { (Fraction of Electrons Accepted })}{\text { (Fraction of Hadrons Not Rejected })} \text {. }
$$

Figure 4 shows a plot of this rejection power for a range of energies in the BSD as a function of electron acceptance. The electron acceptance is based on a threshold signal cut made on the BSD to select a population of particles to analyze. After making this cut we look at how many electron events from the beam run we would cut from the total. As can be seen from Figure 4, if we reduce our electron acceptance we increase our rejection power. This increased sensitivity comes at the cost of the number of total events we can analyze. A detailed description of the BSD CERN test hardware and the results from the run can be found elsewhere [4][5].

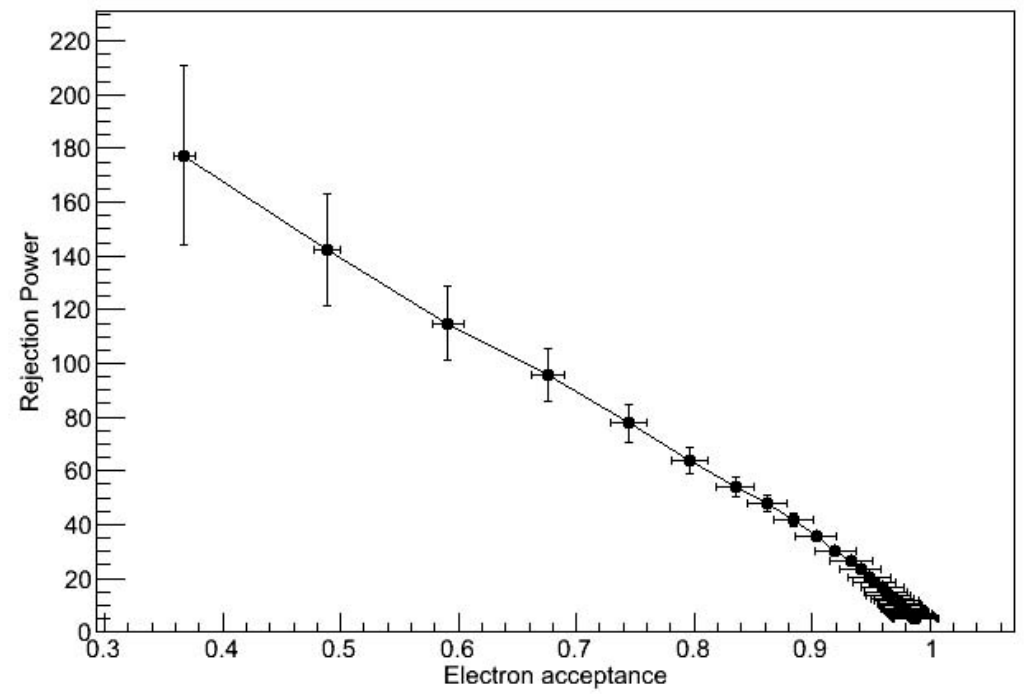

Figure 4: BSD Rejection Power for $100 \mathrm{GeV}-125 \mathrm{GeV}$ electrons and $250 \mathrm{GeV}-350 \mathrm{GeV}$ pions. Data is from the 2012 CERN beam test.

The detector components were also subjected to thermal vacuum qualification and vibration testing as part of our integration. There were no issues or problems uncovered with the design during these tests. It is worth noting that the polyurethane foam used to cushion and protect the boronated scintillator in the detector enclosure dampened all vibration extremely well providing excellent protection to the detector medium.

Prior to delivering the detector for instrument integration we tested the final detector configuration in the lab with muons. The quartet PMTs were set up to run unswitched for this calibration. Two scintillator paddles measuring 9" x 12 " were set 30 " above and below the detector enclosure to form a muon coincidence trigger with geometric acceptance of $77.3 \mathrm{~cm}^{2} \mathrm{sr}$. This trigger was fed into the BSD electronics box as a master trigger signal and muons were 
collected for 12 hours. This data is plotted in Figures 5 and 6 for the low gain and high gain channels, respectively. We see that there is excellent resolution of the muon signal. Using calibration data measured earlier, we find that we are seeing between 30 and 50 photoelectrons per incident muon in each PMT.
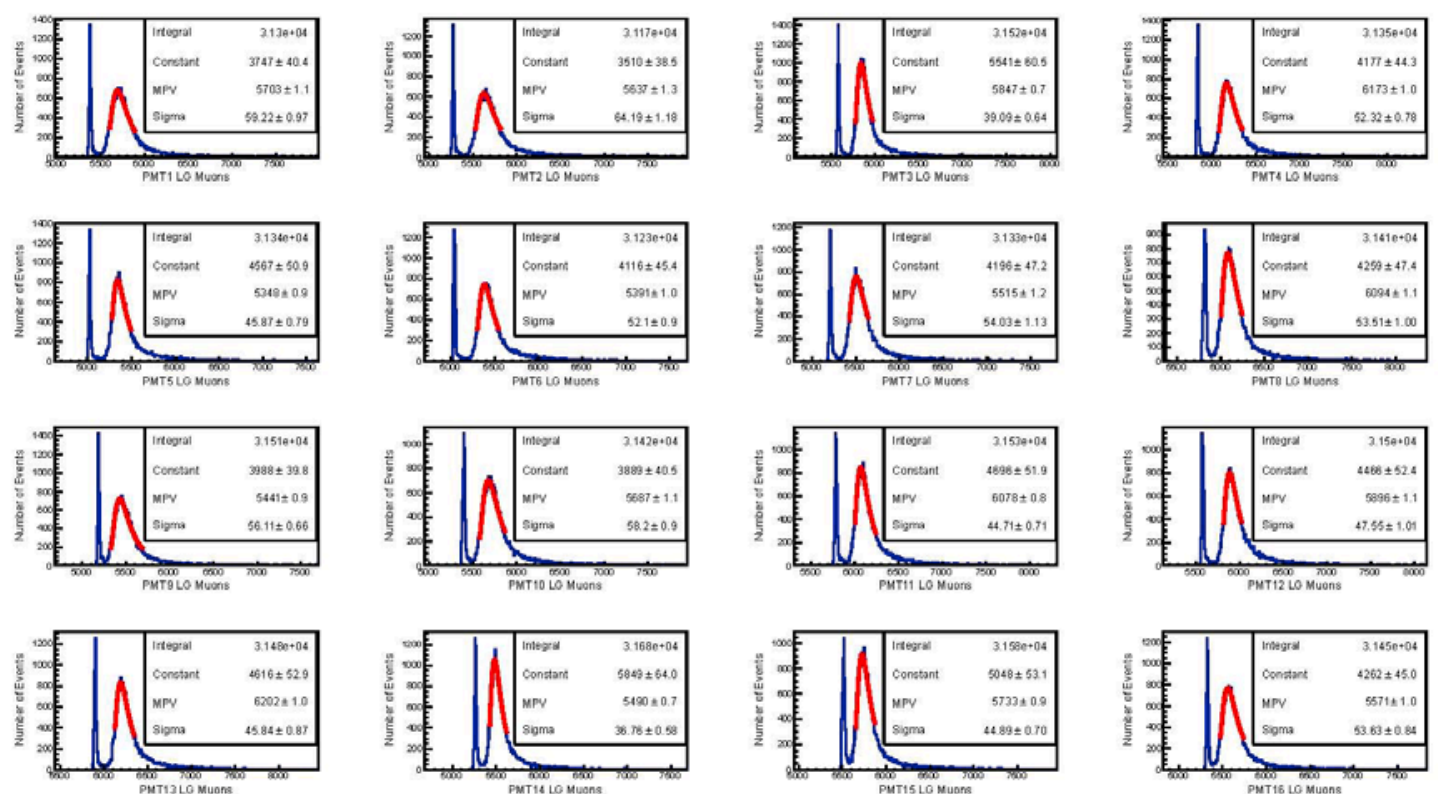

Figure 5: Muon Distribution in the Low Gain FEE channels
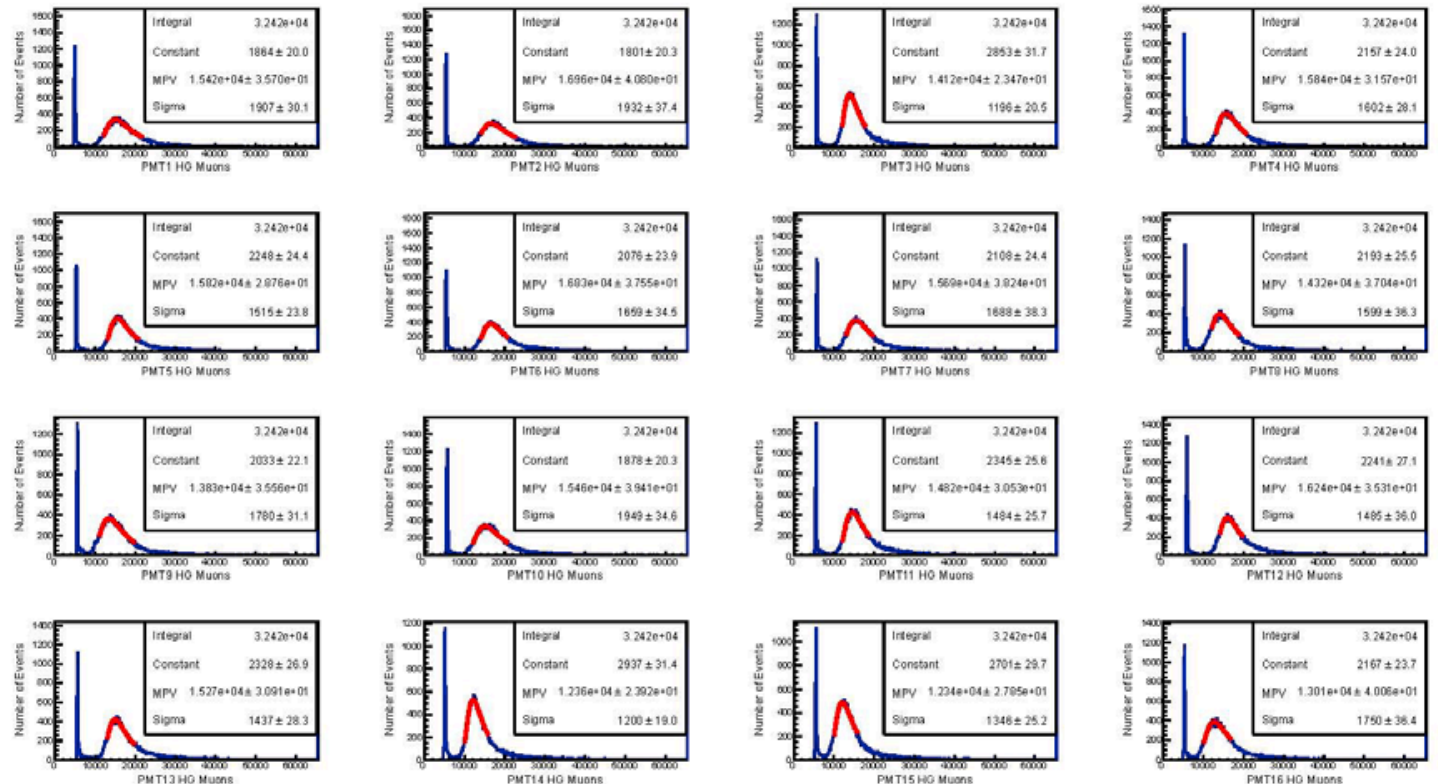

Figure 6: Muon Distribution in the High Gain FEE channels 


\section{Current Status}

The BSD was delivered to Wallops Flight Facility in July of 2014 and integrated onto the instrument bottom plate as shown in Figure 1c. The instrument is currently undergoing environmental testing at NASA Goddard which is expected to complete in August 2015. The BSD has performed extremely well throughout integration and the environmental testing with only minor hardware issues which have been easily addressed.

\section{Acknowledgements}

The ISS-CREAM instrument is supported in the US by NASA grants NNX11AC52G, NNX08AC15G, NNX08AC16G and their predecessor grants as well as by directed RTOP funds to NASA GSFC and WFF. It is supported in Korea by the Creative Research Initiatives of MEST/NRF and by National Research Foundation Grants NRF-2014R1A2A2A01002734 and NRF-2014R1A1A2006456. It is supported in France by IN2P3/CNRS and CNES and in Mexico by DGAPA-UNAM and CONACYT. The BSD instrument team wishes to thank Linda Thompson, Chris Rose, Robin Knight, Peter Patterson, Victor Eyo and the rest of the ISS-CREAM project team for support and guidance during the development, building and integration of the BSD instrument. We also wish to acknowledge and offer our gratitude to the efforts of Kirk Wallace (NKU), Brian Dingee (PSU), Michael Grinsphon (PSU), John Groh (PSU), Zhiyan Wang (PSU), Mark McGinnis (GSFC/SGT), Emanuel Cofie (GSFC/SGT), Jeff Cantwell (GSFC/SGT), Frank San Sebastian (GSFC/SGT), Devin Hahne (GSFC/Bastion), Bert Nahory (GSFC/SGT), Sandy Shuman (GSFC/SGT), Phil Goodwin (GSFC/AS\&D), Ken Simms (GSFC/ADNET) and Holly Hancock (GSFC/ADNET) who contributed in the technical development, design and building of the BSD detector. We could not have delivered the BSD without the insights and contributions of these individuals.

\section{References}

[1] E.S. Seo, et al., Cosmic Ray Energetics and Mass for the International Space Station, Advances in Space Research, 53/10 (2014), 1451-1455.

[2] D.M. Drake, W.C Feldman and C. Hurlbut, New Electronically Black Neutron Detectors, Nuclear Instruments and Methods A, 247 (1986) 576-582.

[3] J.T. Link et al., Scintillation Detector for the Measurement of Ultra-Heavy Cosmic Rays on the Super-TIGER Experiment, Proceedings of the $32^{\text {nd }}$ International Cosmic Ray Conference, Beijing, 2011.

[4] T. Anderson, et al., The ISS-CREAM Boronated Scintillation Detector, Proceedings of the $33^{\text {rd }}$ International Cosmic Ray Conference, Rio de Janeiro, 2013.

[5] T. Anderson, Exploring the Cosmic Ray Spectrum With the CREAM Experiment, PhD Thesis, Penn State University, 2013. 